\title{
Pontos (de vista) sobre o patrimônio: entre o escaneamento e a fotogrametria
}

\author{
Points (of view) about architectural heritage: between scanning and photogrammetry
}

\author{
Adriane Borda Almeida da Silva \\ UFPel, Brasil \\ adribord@hotmail.com \\ Diego Sacco Silveira \\ Triangular Engenharia, Pelotas, Brasil \\ Diego
}

\author{
Alex Medina \\ UFPR, Brasil \\ asmedina@ufpr.br \\ Luisa Félix Dalla Vecchia
UFPel, Brasil
luisafelixd@gmail.com
}

\begin{abstract}
This paper shows the result of a partnership between architects, cartographer and civil engineers, masters and undergraduate students, researchers focused on digital representation technologies applied to architecture, to perform three-dimensional scanning of entire buildings, internally and externally. The study is directed to exploring different types of representation which can be obtained from a point cloud to attribute accessibility to information regarding architectural heritage: the cloud itself, as an accurate record of the current state, form and appearance of the scanned objects; direct visualization in virtual tours; the acquisition of architectural documentation; the representation of appropriate models for 3D printing.
\end{abstract}

Keywords: Representation; architectural heritage; point cloud; scanning; photogrammetry

\section{Introdução}

Este estudo trata de desenvolver habilidades para o uso de tecnologias de aquisição e tratamento de nuvens de pontos para aplicações em representações de patrimônio arquitetônico junto a um contexto específico de ensino, pesquisa e extensão na área de arquitetura.

Nuvens de pontos, neste caso, ficam caracterizadas por um tipo de representação digital constituído por um conjunto de pontos, sobre os quais são conhecidas as suas posições no espaço tridimensional, todas elas relativas a um mesmo sistema de coordenadas. Para este modelo, não existe a definição de linhas, nem de superfícies e nem de sólidos. Entretanto, uma nuvem de pontos pode ser interpretada, visualmente, como representação de qualquer uma destas entidades, caracterizando um objeto tridimensional, por mais complexa que seja a sua geometria. Esta interpretação, visual, pode ser facilitada quando a estes pontos são associados outros parâmetros, como é o caso da cor. Para isto, são atribuídos valores em RGB para cada ponto, podendo assim, o conjunto, corresponder à aparência da superfície representada.

Os processos de geração de uma nuvem de pontos abordados neste estudo dizem respeito ao uso de tecnologias por escaneamento a laser e por fotogrametria.

Especificamente, quanto ao significado que adquirem estas tecnologias para estudos que envolvem a arquitetura de interesse patrimonial, ESTEVE et al (2011) registra: "...el empirismo, intuición e inspiración de la actividad de investigación in situ, del patrimonio edificado, dejará de ser la práctica común, y se dará paso al conocimiento científico y de alta precisión de la geometría de los monumentos" (p.42)
A partir de diversos trabalhos como, por exemplo, Groetelaars e Amorim (2011), Esteve et al (2011), Groetelaars e Amorim (2012), Dezen-Kempter et al (2015), Groetelaars (2015), observa-se o quanto em práticas científicas e profissionais de arquitetura esta cultura está sendo construída.

Embora a infraestrutura conceitual e tecnológica para também estabelecer esta cultura junto ao contexto acadêmico, onde se desenvolve este trabalho, ainda sejam insuficientes, as necessidades geradas junto ao desenvolvimento de projetos, impulsionou a busca por parcerias que proporcionassem usufruir imediatamente das potencialidades de tais tecnologias. Em março deste ano, foi possível promover uma primeira ação de escaneamento a laser na escala de um edifício. Para tanto, constituiu-se uma equipe que envolveu arquitetos, engenheiros cartógrafo e civil, estudantes de mestrado e graduação, pesquisadores focados no tema de tecnologias digitais de representação aplicadas à arquitetura. Esta equipe reúne experiências prévias especificas de investigação, como a descrita em Soria-Medina et al (2013), de prática profissional, de uso de scanner a laser e da fotogrametria na geração de nuvens de pontos, com objetos de diferentes escalas (www.triangularengenharia.com.br) e acadêmicas, relativas às aplicações no âmbito da pesquisa e da extensão universitária, como as referidas em Borda et al (2012) e Félix et al (2015).

O propósito deste estudo conecta objetivos dos projetos MODELA Pelotas e do "Museu do Conhecimento para Todos", desenvolvidos no âmbito da Universidade Federal de Pelotas (UFPel). O primeiro adota as edificações tombadas da cidade de Pelotas como objeto de referência para estudos de representação. $O$ segundo inclui a produção de modelos 
táteis para atribuir acessibilidade à informação sobre a edificação que abriga o próprio Museu (casa-museu). Uma edificação de valor patrimonial para a cidade de Pelotas, de propriedade da UFPel, selecionada para ser escaneada.

\section{O edifício e as representações produzidas}

O Casarão 8 foi construído em 1878, no entorno da praça central da cidade de Pelotas. Uma construção com elementos ecléticos, coroada por frontões curvos e platibanda mista, com recuos para ajardinamento e reconhecida por seu porão alto, como pode ser ilustrado pelas imagens da primeira linha da figura 1. No interior, encontra-se vazia em sua maior parte, porém as salas possuem forros trabalhados em estuque com relevos em gesso feito a mão por artesãos portugueses (Gutierrez,2004 e Chevallier, 2002). Estes relevos em gesso podem ser exemplificados pela primeira imagem da segunda linha da figura 1. E ao lado, nesta mesma linha, tem-se uma estrutura em ferro e vidro que cobre uma claraboia, cuja visão só pode ser facilmente contemplada desde edificações em altura de seu entorno. Memória viva do apogeu econômico da cidade, decorrente da produção de charque, hoje faz parte do conjunto de casarões de Pelotas tombados pelo Instituto do Patrimônio Histórico e Artístico Nacional (IPHAN).

Em trabalhos anteriores, utilizando-se de documentação arquitetônica produzida por métodos de levantamentos tradicionais, deu-se início ao processo de produção de modelos físicos por meio de técnicas de fabricação digital, seja por corte a laser ou impressão 3D. Salvo os detalhes arquitetônicos que envolvem geometrias complexas, como as dos estuques em gesso, os processos empregados, de modelagem digital tradicional, deram conta da obtenção de modelos tridimensionais apropriados para a produção dos modelos táteis. A última linha da figura 1 ilustra os tipos de representações digitais produzidas, com os elementos de fachada simplificados. Tais processos deram conta também de formas de revolução, como pode ser o caso ilustrado pela imagem da direita da segunda coluna da figura 1 , relativa ao modelo da estrutura que cobre a claraboia.

A problemática passou a residir no tempo de produção das representações digitais dos elementos esculturais que caracterizam os adornos da edificação. A oportunidade de aquisição de nuvem de pontos se constituiu como perspectiva de solução. Entretanto, a questão da falta de infraestrutura junto ao contexto em questão se estende para além da disponibilização de um scanner 3D capaz de digitalizar uma edificação. $O$ interesse imediato está na formação para exploração de uso da nuvem de pontos. Observando-se os referenciais anteriormente citados e pela experiência prévia da equipe, avaliou-se necessário identificar, para o contexto em questão, tecnologias e métodos que possam se estabelecer junto ao contexto em questão. Tem-se em vista o propósito de uso de tecnologias de uso gratuito e/ou, na medida do possível, já veiculadas junto às práticas formativas da faculdade de arquitetura que envolve este estudo.
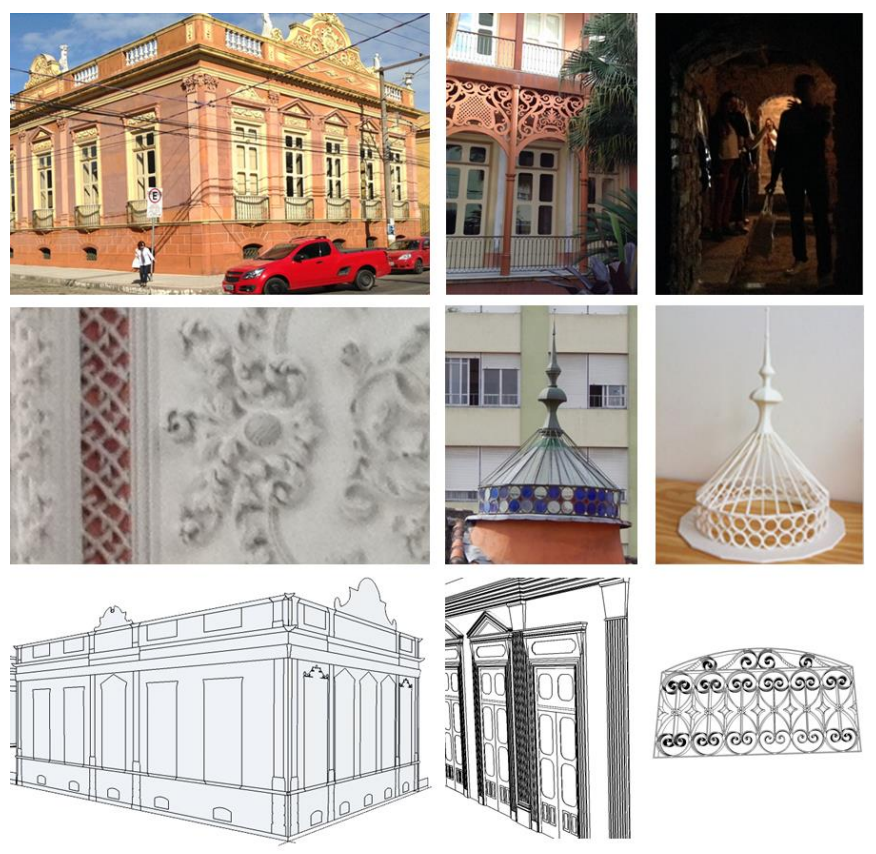

Figura 01: ilustrações sobre a edificação representada. Fonte: fotografias da edificação: autores; fotografia do modelo impresso: Peronti, Veiga e Borda (2016); imagens de modelos digitais: Borges et al (2016),

\section{Procedimentos Metodológicos}

O estudo se estrutura a partir da promoção de momentos de formação/investigação aliados ao propósito de obtenção de produtos que efetivamente respondam às demandas imediatas de representação da edificação.

Foram delimitados três momentos de estudo, sendo cada um deles acompanhado de revisões bibliográficas que permitam o nivelamento e o avanço de conhecimento da equipe envolvida.

1) Planejamento para a aquisição da informação: por meio da nuvem de pontos por escaneamento 3D da edificação e por meio da obtenção de fotos para a fotogrametria em nível de detalhes;

2) Pré-processamento: da nuvem de pontos obtida por escaneamento 3D que compreende a concatenação das nuvens de pontos brutas obtidas pelo laser scanner em cada uma das estações, de forma a obter uma nuvem de pontos única em um mesmo sistema de coordenadas; das fotografias para a obtenção da nuvem de pontos por estereoscopia digital;

3) Processamento: tratamento das nuvens de pontos em softwares específicos para geração de produtos como renderização e animações; documentação arquitetônica (ortofotos, plantas baixas, cortes, fachadas, perspectivas); modelos digitais apropriados para a impressão 3D; modelos para a realidade aumentada.

Deve-se destacar que a primeira etapa, de uso do scanner propriamente dito, esteve prevista exclusivamente para os trabalhos de escaneamento total da edificação, sem o propósito de treinamento de toda a equipe para o uso do equipamento em si, mas de compreensão de como se dá o emprego da técnica. 
O equipamento utilizado para o escaneamento a laser foi um Faro Focus X330, com capacidade até 976.000 pontos por segundo e alcance de $330 \mathrm{~m}$, registrando a informação de cor e tendo câmera integrada. Equipamento de propriedade da Empresa Triangular, Pelotas, RS, Brasil.

\section{Resultados preliminares \\ Sobre o processo de aquisição da nuvem de pontos por laser scanner}

Durante os dias 29, 30 e 31 de março de 2016, deram-se início às atividades de escaneamento a laser. Naturalmente, momentos de nivelamento sobre os conhecimentos próprios de cada uma das especificidades foram acontecendo, abordando: a cartografia; a fotogrametria; as especificações técnicas do equipamento utilizado para o escaneamento; demonstrações das técnicas implementadas pelos softwares envolvidos para o processamento e registro das nuvens de pontos; as limitações tecnológicas para a automatização da obtenção de contornos a partir da nuvem; os formatos obtidos frente às compatibilidades necessárias para a produção de documentação arquitetônica; as interfaces com BIM e os níveis de processamento necessários para a impressão 3D a partir de uma tecnologia específica (aditiva, por deposição de camadas). Desde este momento foram sendo então mapeadas bibliografias de apoio para cada desdobramento no estudo.

Os resultados deste primeiro encontro foram relativos à execução do planejamento, aquisição e pré-processamento de quase a totalidade do primeiro piso do corpo principal da edificação. As duas primeiras imagens da figura 2 referem-se às projeções ortogonais, em vista superior e em perspectiva isométrica, da nuvem de pontos. Sobre a vista superior está também o registro de cada uma das posições utilizadas para localizar o equipamento. Observa-se que dentro de um mesmo cômodo há mais de um local de posicionamento da estação para que o conjunto de cada varredura a laser seja capaz de visualizar todas as superfícies que definem a edificação. Uma prática necessária para a concatenação das nuvens é retratada pela localização da estação sob os marcos das portas. Este espaço de transição entre um ambiente e outro garante que a visão da câmera e consequentemente a abrangência do laser possam registrar os parâmetros dos pontos (RGB e coordenadas) entre os dois ambientes que a porta está situada de maneira redundante. Isto garante precisão no momento de alinhar as nuvens obtidas por cada posição da estação em um único sistema de coordenadas.

Para a constituição da primeira nuvem foram realizadas aproximadamente 24 horas de trabalho, sendo 16 horas de escaneamento e 8 horas de pré-processamento. A configuração da nuvem do primeiro momento compreendeu 53 escaneamentos, cada um com uma média de 10.000.000 pontos. No segundo momento, foram feitos mais 29 escaneamentos, totalizando 82 e aproximadamente 820.000 .000 pontos. Para explicar o significado destas grandezas foram sendo citados casos da experiência profissional, como por exemplo, que uma casa de $500 \mathrm{~m}^{2}$ foi escaneada em sua totalidade com 50 escaneamentos. Ou que um pavimento inteiro de um grande edifício pode levar até 130 escaneamentos, dependendo principalmente do número de ambientes.

A imagem de baixo da figura 2 representa o estágio atual da nuvem de pontos, que não está completa, embora tenha informação suficiente para ser explorada junto ao momento de processamento, o qual está focado nas aplicações.

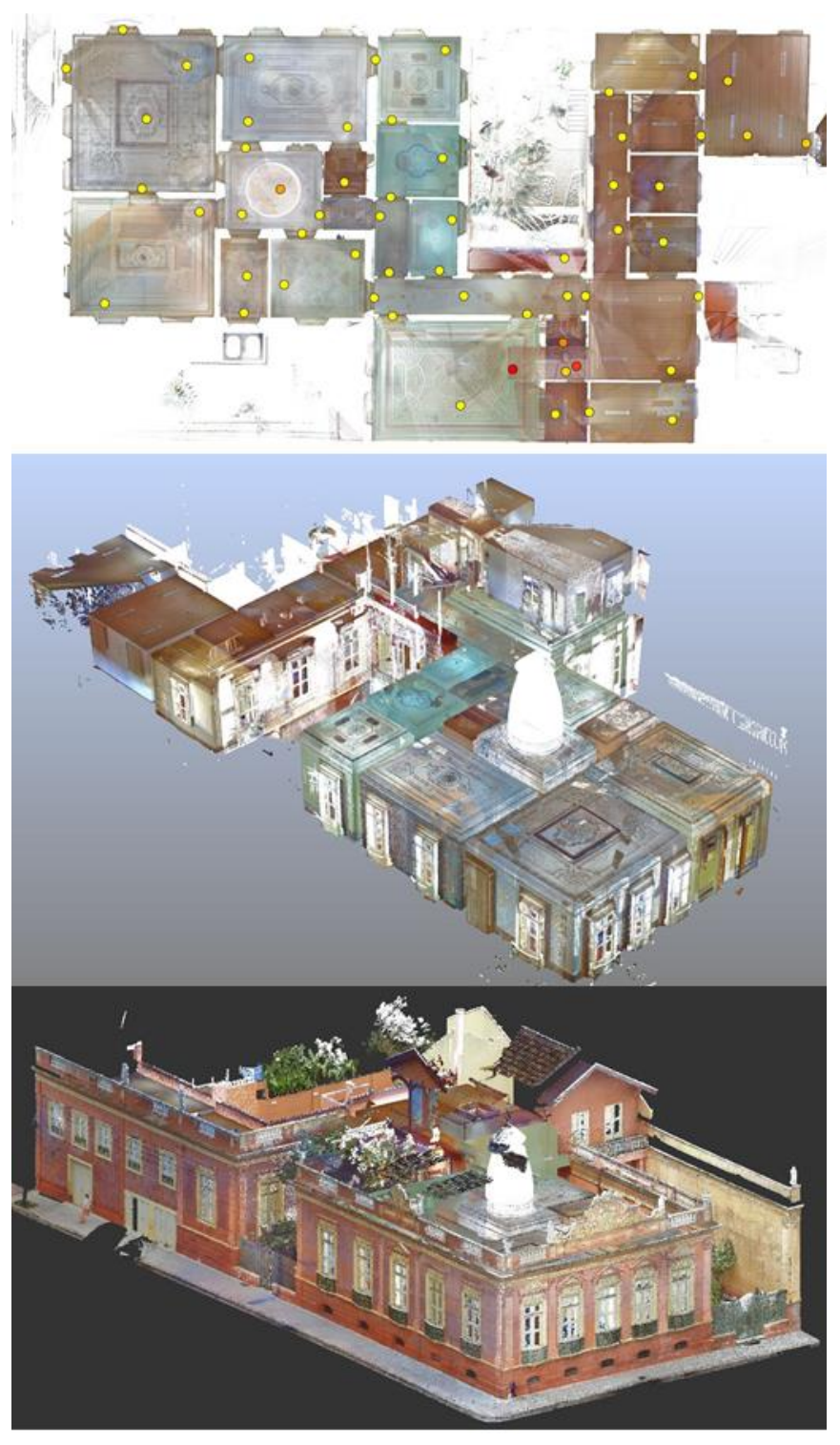

Figura 02: Projeções ortogonais da nuvem de pontos do Casarão 8. Vista superior (com a marcação das posições do equipamento) e perspectivas axonométricas.

Fonte: autores

A atividade incluiu também a proposta de difusão da ação em um contexto acadêmico e profissional mais amplo. Por meio de uma palestra aberta ao público, foi possível contar com profissionais arquitetos, especialistas na área de patrimônio, que auxiliaram a problematizar os processos de 
reconhecimento das potencialidades de tais tecnologias, acesso e apropriação junto ao contexto profissional e acadêmico de arquitetura.

Uma das questões enfatizadas pelos especialistas na aquisição e pré-processamento das nuvens, refere-se ao fato de que estes dados resultam em arquivos densos, de difícil manejo em computadores de uso corrente na prática de arquitetura. Desta maneira, o pré-processamento foi todo realizado nos computadores dos especialistas. Isto compreendeu a atividade de concatenação das nuvens e conseqüentemente o primeiro modelo tridimensional. Isto envolveu a atribuição de valores de RGB a cada ponto, de maneira automatizada, por associação às fotografias obtidas durante a aquisição das nuvens. Todos estes procedimentos foram acompanhados pelos estudantes bolsistas envolvidos nos projetos e em disciplinas de pós-graduação.

\section{Sobre o processo de aquisição da nuvem de pontos a partir de fotogrametria}

O conjunto de imagens da figura 3 ilustra o processo que foi desenvolvido para introduzir os estudos de obtenção de nuvem de pontos a partir de fotogrametria digital.

O exercício foi realizado com uma gárgula, ilustrada pela imagem superior da figura 3. A partir da imagem inferior esquerda é possível compreender como se dá o processo: sequencias de fotografias (no caso 3 filas de 7 fotos) são dispostas de acordo com a posição da câmera (caracterizada pela normal (em preto) ao plano da imagem (em azul)). As diferentes posições permitem calcular, por triangulação, a posição de cada ponto da superfície do objeto fotografado. As duas imagens da direita são projeções ortogonais, em vistas frontais, da gárgula. Aos pontos da nuvem superior estão associados os valores em RGB, fazendo com que se assemelhe a uma fotografia.

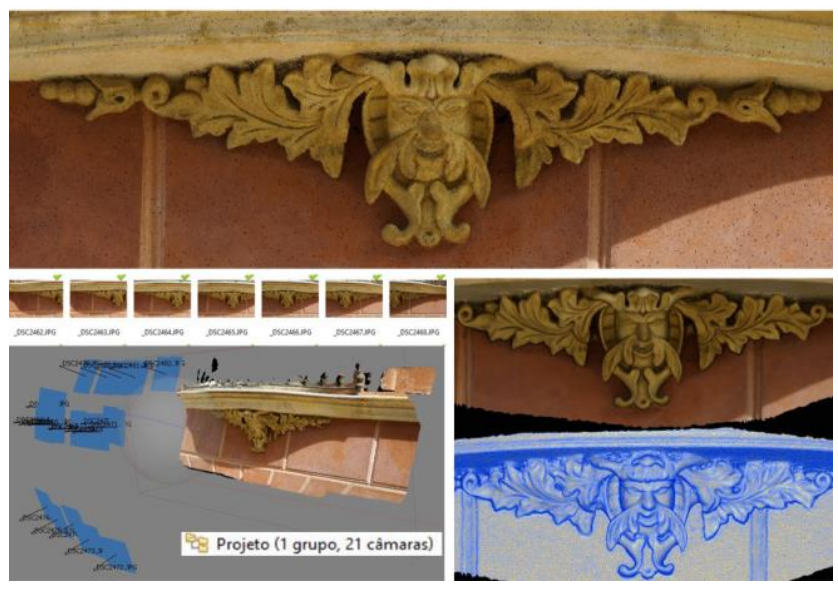

Figura 03: llustrações sobre o processo de aquisição de nuvem de pontos de uma gárgula por fotogrametria. Fonte: Autores
Esta atividade foi desenvolvida paralelamente ao primeiro momento de uso do laser scanner, principalmente para promover comparações entre os dois processos.

A nuvem de pontos desta gárgula, quando gerada pelo scanner empregado, obteve aproximadamente 50.000 pontos. Já a nuvem de pontos adquirida por fotogrametria obteve 1.300 .000 pontos. Deve-se considerar que esta diferença está atribuída às especificações técnicas dos equipamentos. Existem scanners de alta precisão. Mas, o objetivo foi de demonstrar que neste caso, com os equipamentos empregados, consegue-se maior precisão de representação em nível de detalhes, com a fotogrametria.

\section{Sobre a renderização e animação a partir da nuvem}

As imagens da figura 4 foram capturadas de um experimento de produção de um passeio virtual simulado a partir da nuvem de pontos. $O$ estudo se apoia em trabalhos como o apresentado em ScanLab (2012), onde é possível, através da internet, realizar um passeio por uma nuvem de pontos da Galeria de Barcos do Museu de Ciências de Londres. Conforme registrado na publicação referida esta nuvem decorreu de 276 posições do escâner para cobrir todos os detalhes da exposição, compreendendo uma nuvem de mais de 2 bilhões de pontos.

O resultado aqui obtido ainda é incipiente, constituindo-se um grupo de estudos para explorar o desenvolvimento deste tipo de produto. O software utilizado para processamento da nuvem de pontos a fim de se obter o passeio virtual foi 0 Revit Autodesk, por reunir, neste momento, as seguintes características: adequar-se às condições de uso, em sua versão livre para uso educacional; ser compatível e robusto para tratar a nuvem de pontos gerada e a equipe ter um conhecimento introdutório para o uso de tal tecnologia. Os procedimentos realizados foram: importação da nuvem; definição dos planos horizontais de referência (níveis superior e inferior); definição do nível de trajetória da câmera para caracterizar o passeio virtual. Com este primeiro experimento perceberam-se limitações para alterar distância focal, para realizar a troca de nível durante o passeio e para girar a câmera no eixo vertical.

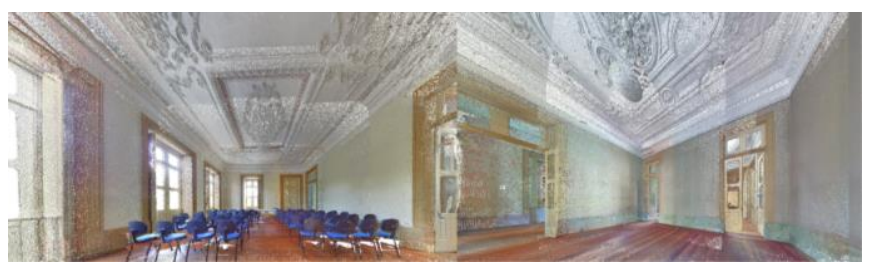

Figura 04: Imagens obtidas a partir do passeio virtual pela nuvem de pontos do Casarão 8. Fonte: Cava, Silveira e Borda, 2016

Os passeios virtuais gerados por nuvens de pontos logicamente não se propõem a imprimir realismo na representação. Para a renderização de uma cena, pressupondo simulações, considerando a interação entre luz e matéria, é necessária a caracterização de superfícies. Fantini (2012) aborda a problemática de utilizar nuvens de 
pontos como base para gerar representações passíveis de serem renderizadas. As ferramentas próprias para animação e interatividade não estão desenhadas para processar tanta informação.

\section{Sobre o processamento da nuvem de pontos para a impressão 3D}

Valendo-se também do modelo da gárgula, ainda junto ao primeiro momento de produção, os especialistas em processamento da nuvem de pontos realizaram a demonstração de como obter um modelo apropriado para a impressão 3D. A ferramenta utilizada foi 3DReshaper 9.1. Foram ressaltados problemas associados ao processo, o qual muitas vezes não se faz totalmente automatizado, exigindo intervenções pontuais para ajustar a configuração da malha triangular que conecta cada um dos pontos da nuvem. Desta maneira, o primeiro passo é a geração de um modelo de superfície. Para a impressão 3D faz-se necessário avançar para a geração de um modelo sólido.

A partir desta introdução, se estabeleceu um grupo específico de estudos para experimentar ferramentas alternativas, tendo em vista que o software utilizado como exemplo é proprietário e não disponível para o contexto acadêmico em questão. Passou-se a experimentar o software MeshLab.

A figura 5 mostra o detalhe de uma porta do Casarão 8 em diferentes escalas. $\mathrm{Na}$ imagem da direita desta figura é possível identificar a malha triangular e sua densidade tendo em vista a consideração de que todos os pontos da nuvem passam a se caracterizar como vértices de uma superfície poliédrica. Faz-se necessário estudar as estratégias para a simplificação da malha que considere o nível de resolução da tecnologia de impressão 3D empregada, para que não se perca informação que descaracterize a geometria do objeto possível de ser representada.

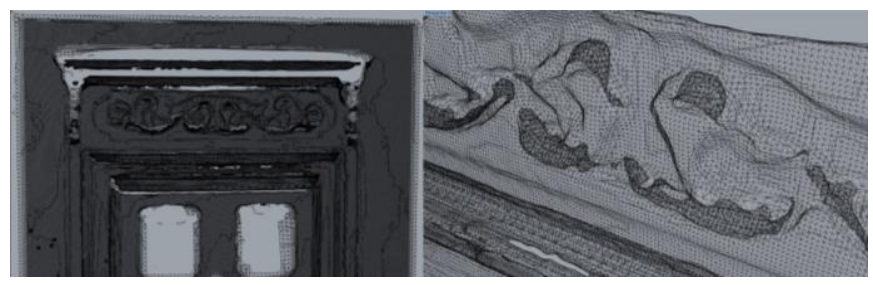

Figura 05: llustrações sobre o processamento da nuvem de pontos para a compatibilização com a tecnologia de impressão 3D. Fonte: Cava, Silveira e Borda, 2016

\section{Sobre o processamento da nuvem de pontos para a documentação arquitetônica}

A figura 6 ilustra os produtos até então adquiridos da nuvem de pontos relativos ao formato tradicional de documentação arquitetônica. A imagem superior esquerda resulta de uma projeção ortogonal, em vista frontal, da nuvem de pontos do Casarão 8. Ao lado tem-se a mesma vista obtida por processos tradicionais de levantamento. As linhas sobrepostas, em vermelho, destacam as diferenças detectadas entre as duas representações. Em Parker et al (2015) realizaram-se análises gráficas para a compreensão de lógicas compositivas da edificação em questão utilizandose da documentação arquitetônica disponibilizada anteriormente. Observam-se diferenças significativas que comprometem as hipóteses inicialmente elaboradas.

A planta baixa, na figura 3, também está sendo problematizada frente à documentação tradicional.

Por outra parte, constitui-se um grupo específico para investir na conexão de nuvens de ponto com tecnologias BIM.

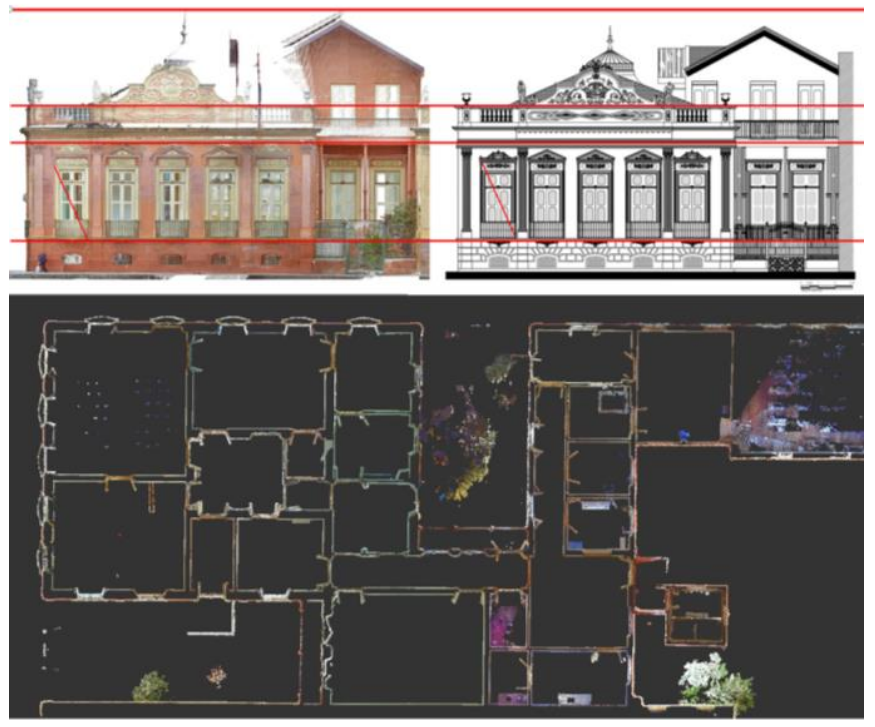

Figura 06: Documentação arquitetônica adquirida a partir de nuvem de pontos e comparação com a documentação tradicional. Fonte: Autores

\section{Discussão}

Neste estudo tratou-se essencialmente do problema de construir uma cultura, em um contexto acadêmico específico, capaz de perceber a importância e de usufruir imediatamente das potencialidades de uma nuvem de pontos para a representação de uma edificação de interesse patrimonial. Sem uma infraestrutura conceitual e tecnológica suficiente para isto, buscou-se o estabelecimento de uma parceria.

O método empregado está permitindo estruturar uma pauta de investigação para cada um dos temas que envolvem a caracterização dos produtos que estão sendo gerados.

O estudo, embora com resultados concretos, já disponibilizando as nuvens de pontos para serem visualizadas e possibilitarem explorações e passeios virtuais, encontra-se em desenvolvimento. 


\section{Conclusões}

A ação de digitalização tridimensional do Casarão 8 se constitui, para o contexto em que se insere, uma ação inovadora e que está permitindo transformar efetivamente 0 processo de documentação arquitetônica. Desta maneira, deixa de ser a prática comum, dando passo ao conhecimento científico e de alta precisão da geometria do patrimônio representado, como anunciado anteriormente junto à bibliografia utilizada. $\mathrm{E}$, isto só se tornou possível pela agregação de diferentes pontos de vista, sob a abordagem científica, profissional e educativa.

\section{Agradecimentos}

Aos programas de pós graduação em Memória e Patrimônio e ao de Arquitetura e Urbanismo, ambos da Universidade Federal de Pelotas, os quais subsidiaram a presença do Prof. Dr, Alex Medina para o estabelecimento da parceria junto ao GEGRADI.

À Livia Cava, estudante FAURB/UFPel que tem contribuído com o aprendizado em BIM adquirido junto à Savannah College of Art and Design, Programa Ciências sem Fronteiras.

À empresa Triangular Engenharia que oportunizou, de maneira gratuita, o emprego da tecnologia assim como todo o acompanhamento especializado.

Aos órgãos e programas de fomento CNPq, FAPERGS, Programa ALFA III e PROEXT, por permitirem uma infraestrutura mínima para o desenvolvimento dos estudos, desde bolsas de iniciação científica e tecnológica, aos equipamentos, como impressora 3D.

\section{Referências}

CAVA, Livia; SILVEIRA, Diego; BORDA, Adriane. Tratamento de nuvem de pontos para a impressão 3D: entre o estado da arte e as tecnologias disponíveis em um contexto específico de formação de arquitetura. In Anais do CIC2016/UPEL

BORGES, G.; SILVEIRA, D.; BORDA, A.; JANTZEN, S. Leituras sobre o Casarão 8 apoiadas em tecnologias avançadas de representação: um trânsito entre estética, iconografia, geometria e técnicas construtivas. In: 2 을 SIEPE Semana Integrada de ensino, pesquisa e extensão. $25^{\circ} \mathrm{CIC}$ Congresso de Iniciação Científica da UFPel. Pelotas: UFPel, 2016. v.1. p.14

DEZEN-KEMPTER, E.; SOIBELMAN, L.; CHEN, M.; MÜLLER, A.V. Escaneamento 3D a laser, fotogrametria e modelagem da informação da construção para gestão e operação de edificações históricas. Gestão e Tecnologia de Projetos, São Paulo, v. 10, n. 2, p. 113-124, jul./dez. 2015 http://dx.doi.org/10.11606/gtp.v10i2.102710

ESTEVE, P. N.; BOQUERA, J. H.; FLORES, A. M.; COSTA, H. B.; RíOS, J. L. D. Aplicaciones de la tecnología de digitalización tridimensional por la coordinación de monumentos históricos del instituto nacional de arqueología e historia (INAH) en México df. (2009 y 2010). // Revista de EGA;2011, Issue 17, p42

FANTINI, F. Modelos con nivel de detalle variable realizados mediante un levantamiento digital aplicados a la arqueologia. EGA. Revista de Expresión Gráfica Arquitectónica, [S.I.], n. 19, p. 306-317, mar. 2012. Disponível <http://polipapers.upv.es/index.php/EGA/article/view/1383>. Acessado em 20/05/2016.

GROETELAARS, N. J. Criação de modleos BIM a partir de "nuvens de pontos": estudos de métodos e técnicas para a documentação arquitetônica. Tese (Doutorado) Universidade Federal da Bahia, Faculdade de Arquitetura; 2015.

GROETELAARS, N. J.; AMORIM, A. L. Tecnologia 3D Laser Scanning: características, processos e ferramentas para manipulação de nuvens de pontos. SIGRADI 2011

GROETELAARS, N. J.; AMORIM, A. L. Um panorama sobre o uso de nuvens de pontos para criação de modelos BIM. In: Seminário Nacional de Documentação do Patrimônio Arquitetônico com o uso de tecnologias digitais, 2., 2012, Belém. Anais... Belém: UFPA, 2012.

PARKER, G.; BORGES, L. F.; Peronti, Gabriela; BORDA,A.B.A.S. Análises geométricas do casarão 8: compreensão histórica, projetual e construtiva In: SIEPE Semana Integrada de Ensino, pesquisa e extensão, 2015, Pelotas. XXIII CIC Congresso de Iniciação Científica da UFPel. Pelotas: UFPel, 2015. v.1. p.14

PERONTI, G.; VEIGA, M.; BORDA, A. A extensão da percepção: uma experiência com a produção de modelos táteis que descrevem a ambiência gerada pela cúpula do Casarão 8, Pelotas, RS. In: $2^{\circ}$ SIEPE Semana Integrada de ensino, pesquisa e extensão. $3^{\circ}$ CEC Congresso de Extensão e Cultura da UFPel. Pelotas: UFPel, 2016. v.1. p.14

SORIA-MEDINA, A.; MARTINEZ, J.; ARIAS, P.; ARMESTO, J.; BUFFARAANTUNES, A. Z. Three-Dimensional Modeling of the Romanesque Church of Santa Maria de Castrelos (Vigo - Spain) Using Terrestrial Laser Scanner. ISPRS - International Archives of the Photogrammetry, Remote Sensing and Spatial Information Sciences, v. XL-5/W2, p. 601-604, jul. 2013. DOI: 10.5194/isprsarchives-XL-5-W2-601-2013.

ScanLab 2012. http://scanlabprojects.co.uk/projects/sciencemuseum 